\title{
Les ambiguïtés du colonialisme dans The Tempest
}

Jean-Pierre Villquin

\section{Q OpenEdition \\ Journals}

\section{Édition électronique}

URL : http://journals.openedition.org/shakespeare/1270

DOI : 10.4000/shakespeare.1270

ISSN : 2271-6424

Éditeur

Société Française Shakespeare

Édition imprimée

Date de publication : 1 novembre 1994

Pagination : 35-56

Référence électronique

Jean-Pierre Villquin, "Les ambiguïtés du colonialisme dans The Tempest ", Actes des congrès de la Société française Shakespeare [En ligne], 12 | 1994, mis en ligne le 01 janvier 2007, consulté le 04 mai 2019. URL : http://journals.openedition.org/shakespeare/1270 ; DOI : 10.4000/shakespeare.1270 
COSMOPOLITISME ET INSULARITÉ 


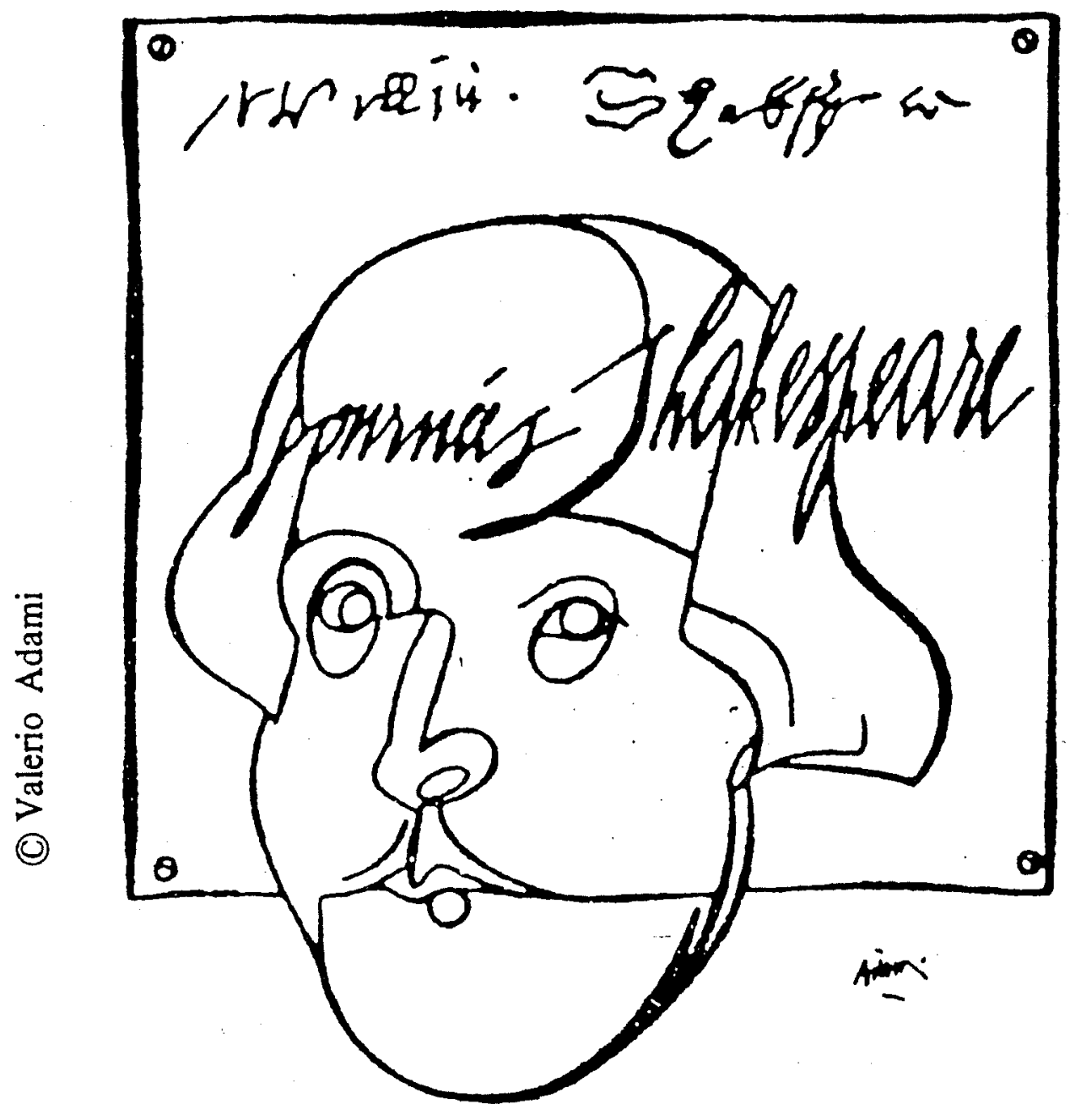

Affiche de Valerio Adami, spécialement conçue pour les journées Shakespeare 1979 


\section{SHAKESPEARE}

COSMOPOLITISME ET INSULARITÉ

Société Française Shakespeare

Actes du Congrès 1993

sous la direction

de

M. T. JONES-DAVIES

Ouvrage publié avec le soutien de

BARCLAYS

BARCLAYS BANK S.A

PARIS

LES BELLES LETTRES

1994 
Tous droits de traduction, de reproduction et d'adaptation réservés pour tous les pays.

(C) 1994 Société d'édition Les Belles Lettres, 95 bd Raspail 75006 Paris

ISBN 2.251.69122-7 


\section{Les ambiguïtés du colonialisme dans The Tempest}

L'île de Prospero, quelque part entre Tunis et Naples, n'est ni une colonie ni un paradis perdu au milieu de la Méditerranée ; c'était une terre d'exil pour Sycorax, c'est un refuge pour les naufragés, et sera aussi le creuset, le microcosme du meilleur des mondes. La notion de premier occupant est mise en question dès le début. Caliban, ce cannibale si attachant qui revendique la possession d'un royaume dérisoire est un immigré de la deuxième génération, fils d'une sorcière expulsée de la terre d'Afrique. Prospero, lui, a échoué là par hasard et n'a nullement l'intention de peupler l'île de colons, pas plus qu'il n'a l'intention d'en exploiter les ressources à des fins mercantiles. Nous avons là l'archétype du colon dominateur, celui du sauvage rebelle et paresseux, et celui de la colonie aux ressources inépuisables ; cependant Prospero retournera chez lui les mains vides, riche d'une expérience équivoque, Caliban retrouvera son état naturel, et l'île restera une utopie. Tout est donc affaire de perspectives qui sont la source des ambiguités. The Tempest peut être considérée comme la vision idéalisée de l'impérialisme anglais qui arrache les indigènes à leurs superstitions, à leurs tabous et à leur sauvagerie, pour les amener, soumis, à apprécier les bienfaits du monde civilisé ${ }^{1}$. C'est une perspective historique qui s'appuie sur les sources et sur les préférences explicites ou implicites du texte, mais les ambiguités apparaissent d'emblée. D'une part le succès de l'opération s'avère douteux, d'autre part notre approche du colonialisme n'est pas ce qu'elle pouvait être au début du dix-septième siècle, et enfin les récits qui ont pu inspirer la pièce sont porteurs de leur propre antidote avec l'exotisme, le merveilleux et le miraculeux qu'ils charient. Dans une première partie, j'essaierai donc d'analyser cet esprit colonial qui agite une époque et qui inspire Shakespeare. Ce dernier nous invite toujours à passer de l'autre côté du miroir, et n'oublie pas de donner la parole aux 'colonisés', Ariel et Caliban. Ariel change simplement de maître, collaborateur par pragmatisme et agent du néo-colonialisme, il n'appartient pas à la race des humains. Caliban, symbole de la vie naturelle et de l'instinct, s'oppose au monde civilisé. Il est peut-être le reflet de la vision qu'on pouvait avoir d'un sauvage 
au temps de Shakespeare, mais il est aussi la projection de ce qu'il y a d'inavouable et d'inacceptable dans le cœur de l'occidental policé, représenté par Prospero ${ }^{2}$. Le 'cannibale' bouc émissaire n'est qu'un aspect des rapports difficiles avec l'Autre, avec l'Étranger, sources de conflits qui, avec le Maure ou le Juif, avaient déjà intéressé Shakespeare pour leur potentialité dramatique. Les facettes et les interprétations sont donc multiples. Dans une seconde partie je ne retiendrai que l'esprit de liberté et de rébellion, ainsi que la notion de nature ou d'instinct quand elle s'oppose à celle de civilisation. Certains critiques voient The Tempest comme un acte politique, une réflexion sur le pouvoir et l'idéologie : la guerre civile de Milan serait alors une image miniaturisée des grandes manœuvres transatlantiques, l'histoire d'amour entre Miranda et Ferdinand serait une ruse politique de Prospero pour assurer son retour au pouvoir, et le désir de Caliban pour Miranda serait une métaphore inversée des conquêtes territoriales ${ }^{3}$. Je serais tenté, au contraire, de voir dans The Tempest une habile et magistrale manipulation : celle de Prospero, agent de Shakespeare, le seul et véritable magicien. Prospero et Caliban sont l'avers et l'envers d'une même monnaie, c'est pourquoi, dans la troisième partie de cette approche des ambiguités du colonialisme, je souhaite mettre en relief ce que je crois être la réhabilitation de l'état naturel, ou tout au moins l'acceptation du monde de l'Autre et du monde de l'instinct, ce qui revient à mettre en question certaines valeurs du monde qui s'enorgueillissait et s'enorgueillit encore d'être civilisé.

Les hurlements du vent dans les haubans, les voiles qui se déchirent, les mâts qui craquent, les coques qui se brisent, marins et voyageurs qui roulent dans l'eau salée, c'est la tempête, phénomène naturel qui, ici, n'est pas seulement le titre et la première scène d'une pièce, car elle en est aussi la métaphore. Elle est le symbole de la fortune de mer, avec ses risques et ses espoirs. The Tempest est émaillée de références et d'allusions aux hasards de la navigation, aux activités des marchands aventuriers et au monde merveilleux et dangereux des colonies. Pour réconforter le roi Alonso qui croit avoir perdu son fils Ferdinand, Gonzalo tente de le consoler en lui rappelant que c'est là peine commune : 


\author{
... Our hint of woe \\ Is common; every day, some sailor's wife, \\ The masters of some merchant, and the merchant, \\ Have just our theme of woe ; $\quad$ (II. i. 3-6)
}

Plus loin, le même Gonzalo évoque les spéculateurs qui attendent d'une expédition un rapport de cinq cents pour cent (III.iii.48). The Tempest fut écrite un ou deux ans après la fondation de Jamestown en Virginie (1609), mais les colonies ou "plantations" faisaient la une de l'actualité dès le seizième siècle, le souverain et les courtisans étaient souvent parties prenantes dans ces expéditions: Cranmer, dans Henry VIII, fait l'éloge de la reine et souligne «la gloire et la grandeur de son nom» qui «régneront pour fonder de nouvelles nations» (V.v.51-2). Mais au-delà du topique et de l'actualité, Shakespeare, comme ses contemporains, est sans doute passionné par les mondes merveilleux, magiques et terrifiants dont les descriptions, dans les nombreux récits et témoignages de voyageurs, nourrissent l'imagination. Gonzalo est prêt à raconter à son retour ce qu'il a vu (III.iii.26). Dès qu'il aperçoit Caliban, Stephano pense à ces "sauvages", à ces "Indiens" qu'il pourrait montrer sur une place publique pour en tirer profit, ou qui feraient un présent digne d'un empereur (II.ii.58 et 68).

Les voyages n'inspirent pas seulement les narrateurs, mais souvent, à travers eux, ils inspirent les dramaturges. Les voyages des frères Shirley en sont un exemple ${ }^{5}$. La simple découverte et l'exploration de lointaines contrées se transforme vite en conquête, conséquence des intérêts économiques et des nécessités ou de l'opportunisme politique. Les rapports entre le nouveau et l'ancien monde, entre l'homme "sauvage" et l'homme "civilisé" suscitent des réactions, des réflexions, des prises de position morales et philosophiques dont on retrouve l'écho dans The Tempest. Tantôt c'est Montaigne dont on reconnaît les mots dans la bouche de Gonzalo, tantôt c'est Sir Francis Bacon qui, un peu plus tard, jettera sur les colonies un regard d'où l'exotisme et le romanesque auront en grande partie disparu : «j'aime à voir coloniser un sol vierge,» écrit-il, «j'entends où les gens n'ont pas été déplantés pour en implanter d'autres ; sinon, c'est une usurpation plutôt qu'une colonie. ${ }^{6}$ Cet essai ne fut publié qu'en 1625 , et en quinze ans les idées avaient sans doute évolué.

Les récits et les documents qui ont pu inspirer la pièce non seulement tissent la trame narrative, mais aussi reflètent les idéo- 
logies du temps. Nous ne retiendrons ici que trois sources parmi les plus révélatrices. La première est l'ouvrage de Sylvester Jourda(i)n, (A) Discovery of the Bermudas Otherwise call'd the Isle of Devils (oct. 1610). Jourdain était du voyage, et fait un rapport circonstancié de l'aventure de cette flotille de neuf bateaux partis de Plymouth le 2 mai 1609 pour la Virginie, commandée par Sir Thomas Gates, Sir Georges Somers et le capitaine Newport. Les navires furent dispersés par une terrible tempête en juillet de cette même année, et le navire amiral, The Adventurer, se brisa sur les côtes des Bermudes. Shakespeare reprend les termes mêmes du récit de Jourdain dans les dialogues des marins qui racontent leur naufrage (V.i.232 et seq.). L'allusion aux «Bermudes toujours troublées par les tempêtes» (I.ii.226) a lancé de nombreux critiques sur la piste des Indiens et des tumultueux rapports entre l'Angleterre et l'Amérique. Ariel évoque le jour où Prospero lui demanda d'aller chercher «la rosée des Bermudes», ce qui est, je pense, une simple image qui n'a apparemment aucun rapport avec le lieu du drame. En effet, dans The Tempest, la flotille venait de Tunis, et, à part le vaisseau d'Alonso, mis à l'abri dans une crique, les autres sont éparpillés en Méditerranée et ensuite cinglent vers Naples (I.ii.236).

Un deuxième document auquel Shakespeare a pu avoir accès est True Declaration of the State of the Colonie in Virginia, inscrit au Registre des Libraires le 8 novembre 1610, rapport du Conseil de la Colonie de Virginie, "dont l'objet", écrit Henri Fluchère, «est moins de raconter l'événement que d'en tirer, ainsi que des conséquences du voyage et du naufrage, comme une morale de la Providence. ${ }^{7}$ En effet, sans anticiper sur ce que nous verrons plus loin, chroniqueurs et voyageurs veulent souvent trouver un sens aux aventures, "chaque événement s'inscrit dans l'ordre et le sens de la volonté supérieure.» ${ }^{8}$ Le théâtre ne faillit pas à cette règle, et Shakespeare inscrit lui aussi l'aventure dans le mythe : l'exil de Prospero est la conséquence de son éloignement volontaire, peut-être de son désintérêt du pouvoir, et le naufrage d'Alonso est la sanction qui lui est infligée pour avoir marié sa fille à un Africain.

Un troisième document auquel Shakespeare a pu avoir accès, est la lettre de William Strachey, A True Repertory of the Wracke (and Redemption of Sir Thomas Gates, Knight). Bien qu'elle ne fût publiée que tardivement, en 1625, par Samuel Purchas $^{9}$, cette lettre date du 15 juillet 1610 , et Shakespeare a pu 
en avoir connaissance grâce à son ami Thomas Russell ${ }^{10}$ dont le gendre, Sir Dudley Digges, faisait partie du Conseil de la Compagnie de Virginie. D'autres sources apportent à la pièce des détails ou des thèmes qui renvoient au monde des colonies : ainsi Setebos, invoqué par Caliban (I.ii.373 et V.i.261), est un dieu de Patagonie selon Drake, et on trouve son nom dans History of Travayle (1577) de Richard Eden, le nom d'Ariel vient des textes ésotériques hébreux, le nom de Caliban est l'anagramme de cannibale ${ }^{11}$. L'un des Essais de Montaigne, traduit en anglais par Florio en 1603, "Les Cannibales", inspire Gonzalo (II.i.139 et seq.) au point qu'il reprend presque mot pour mot le texte de Montaigne: «C'est une nation [...] en laquelle il n'y a aucun espèce de trafique [...] nul cognoissance de lettres; nulle science de nombres; nul nom de magistrat, ny de supériorité politique ; nul usage de service, de richesses ou de pauvreté ; nuls contrats ; nulles successions; nuls partages; nulles occupations qu'oysives [...]» ${ }^{12}$. Comparons ce passage avec le discours de Gonzalo :

I'th' commonwealth I would by contraires

Execute all things; for no kind of trafic

Would I admit; no name of magistrate ;

Letters should not be known; riches, poverty,

And use of service, none ; contract, succession,

Bourn, bound of land, tilth, vineyard, none ;

No use of metal, corn, or wine, or oil ;

No ocupation; all men idle, all ;

And women too, but innocent and pure :

No sovereignty ...

(II.i.143-152)

Entre l'idéalisme utopique de Gonzalo, héritier de Montaigne, et la cruelle servitude dans laquelle est jeté Caliban, il y a une subtile dialectique qui s'est d'ailleurs poursuivie jusqu'au dix-neuvième siècle et même au-delà. Entre les ambiguïtés de l'entente cordiale d'un Bunyan et d'un Defoe avec les bons sauvages, et la manière forte d'un Cecil Rhodes avec les rois Zoulous, on trouve l'expression d'un modus vivendi prudent avec Francis Bacon qui suggère que : «si vous colonisez en un lieu où il y a des sauvages, ne vous contentez pas de les amuser avec des crécelles et des babioles, mais traitez-les avec justice et bienveillance, tout en restant circonspect. ${ }^{13}$ Prospero offre le portrait type du colon blanc aggravé ou contrasté, selon les perspectives, par celui de Trinculo et de Stephano. Bien qu'il échoue par hasard sur l'île, Prospero se 
comporte en conquérant, il détruit l'ordre ancien, supprime les superstitions, et joue au maître d'école avec Caliban comme avec sa fille. Il veut apporter la civilisaion, sa civilisation, pour la substituer aux forces des ténèbres, à l'ignorance, à l'esprit primitif représentés par Sycorax. Il se présente comme un libérateur, délivrant Ariel condamné par Sycorax, parce qu'il était de nature trop délicate pour exécuter les ordres de la sorcière. Mais la liberté d'Ariel est si conditionnelle qu'elle n'est rien d'autre qu'une nouvelle servitude. Prospero se comporte plus ou moins comme le colon qui utilise et exploite ceux qui peuvent le servir, ceux qui créent les richesses, ceux dont on encourage la multiplication pour avoir une main-d'œuvre bon marché ${ }^{14}$. Cette conquête s'effectue par la force et la contrainte, la sorcellerie blanche s'est substituée à la magie noire de Sycorax. Prospero est un nouveau tyran dont les livres ont remplacé les canons, mais il ne règne sur ses sujets que par la terreur, promettant à Ariel une liberté aléatoire, et poursuivant Caliban avec ses "tontons macoutes" polymorphes. Ariel libéré et pourtant asservi, et Caliban asservi et jouissant malgré tout d'une certaine liberté naturelle, sont les deux symboles antithétiques du pouvoir équivoque de ce colon magicien.

Prospero cultive aussi le complexe de supériorité du colon, il semble vouloir transférer le pouvoir qu'il a perdu à Milan sur une île sans sujets ou presque. Cette supériorité, il la doit principalement aux livres de magie que Gonzalo avait placés sur l'esquif qui l'a emporté jusqu'à l'endroit où il a échoué sur l'île. Cette supériorité se manifeste par un sens aigu de la propriété qui pousse Prospero à se vouloir maître, non seulement de "son" esprit, Ariel, mais aussi de ce demi-démon qui se métamorphoses en simple objet, en «cette chose des ténèbres qu'il revendique comme sienne» jusquà la fin ${ }^{15}$. Le désir de possession se double d'un paternalisme qui sera finalement peu apprécié. Toutes les tentatives d'éducation entreprises par Prospero se transforment en échec. Du langage, Caliban retient surtout l'art de proférer des jurons (I.ii.364), il est loin d'être stupide puisqu'il a appris à parler avec la même rapidité que Vendredi dans Robinson Crusoé, mais il est réfractaire à toute notion du bien et du mal, tout au moins dans la perspective du blanc occidental, et par conséquent, l'éducation qu'il reçoit est détournée et ne fait qu'aggraver ses capacités de malfaisance :

A devil, a born devil, on whose nature

Nurture can never stick; on whom my pains,

Humanely taken, all, all lost, quite lost ; 
And as with age his body uglier grows,

So his mind cankers. I will plague them all,

Even to roaring.

(IV.i.188-93)

Cet échec était inscrit dans l'ordre des choses puisqu'il s'agit de la nature même de Caliban, et de sa race, encore faut-il prendre le terme avec précaution, sans compter que la perspective est différente selon que la réplique est attribuée tantôt à Prospero, tantôt à Miranda :

... Abhorred slave,

Which any print of goodness wilt not take,

Being capable of all ill ! I pitied thee,

Took pains to make thee speak, taught thee each hour

One thing or other: when thou didst not, savage,

Know thine own meaning, but wouldst gabble like

A thing most brutish, I endow'd thy purposes

With words that made them known. But thy vile race,

Though thou didst learn, had that in't which good natures

Could not abide to be with; therefore wast thou

Deservedly confin'd into this rock,

Who hadst deserv'd more than a prison.

Prospero passe de l'amère mais simple déception à un racisme actif de l'instant où Caliban jette un regard concupiscent sur la blanche Miranda. Caliban avait eu le tort de croire qu'il pouvait aller jusqu'au bout de la logique d'intégration commencée par Prospero. Le rejet et l'exclusion sont à la hauteur de ce que ce dernier croit être de la générosité :

... Thou most lying slave,

Whom stripes may move, not kindness ! I have us'd thee, Filth as thou art, with human care ; and lodg'd thee

In mine own cell, till thou didst seek to violate

The honour of my child.

C'est l'occasion ou l'alibi pour maltraiter cet "esclave détesté" (I.ii.353), et pour le confiner dans une grotte loin de la demeure du maître. Prospero semble regretter de n'avoir pas pratiqué plus tôt cette politique d'apartheid. Si la force et la loi de Prospero se manifestent par personne interposée, c'est-à-dire par Ariel, si les Bibles de Robinson Crusoe sont ici des livres de magie, la panoplie du parfait colon est au complet, avec quelques flacons d'alcool et un tonneau de Xérès qui assurent la soumission absolue de Caliban. Les goûts changent, les moyens demeurent, puisqu'un siècle plus 
tard, c'est le rhum des Antilles que Robinson Crusoé apprécie et fera apprécier à Vendredi. Quand Caliban, écrasé et vaincu supplie qu'on lui pardonne d'avoir comploté contre la vie de Prospero, et quand il confesse qu'il a été stupide d'avoir cru en Stephano : «âne, trois fois âne d'avoir pris cet ivrogne pour un dieu», Prospero le renvoie d'un geste méprisant. L'attitude et le point de vue de Prospero ne sont ni très subtils ni très nuancés, mais ils sont caractéristiques d'un certain esprit impérialiste. Prospero donnera encore une fois la preuve de son insensibilité quand il restera de marbre devant la souffrance physique et morale de ses victimes (Alonso et Gonzalo) «qui tireraient des larmes à Ariel, cet être non-humain, s'il pouvait pleurer». Après ce tableau sommaire de l'impérialisme anglais tel que nous le renvoie The Tempest, tentons de poursuivre notre analyse en abordant le problème de la liberté et de la servitude d'une part, et celui de la civilisation et de l'instinct d'autre part.

La conscience de la liberté peut se définir par la conscience de la servitude. Ariel, "esprit" libre n'a été délivré du piège de Sycorax que pour être l'otage de Prospero, et le pin dans lequel il était prisonnier risque à tout moment de devenir un chêne. Ariel revendique constamment sa liberté, et finit par l'obtenir au prix de ce qu'on pourrait appeler une véritable collaboration puisqu'il persécute l'indigène Caliban, en le pinçant, et en le soumettant à toutes sortes d'exactions, et cela sur ordre de son supérieur, l'occupant. Caliban, comme Ariel reconnaît la supériorité de Prospero, même si elle se manifeste par l'intermédiaire de la magie. Caliban est fasciné par la puissance, par la maîtrise et par l'autorité de Prospero : «Il faut obéir, car son art est si puissant que du dieu de ma mère, de Sétébos même, il faire son vassal.» (I.ii.273) ${ }^{16}$. Caliban se soumet à la force, mais il revendique son droit de premier occupant et rappelle que cette île est à lui : «elle est à moi par Sycorax ma mère, cette île que vous me prenez.» (I.ii.332). Pourtant tout semble avoir commencé sous les meilleurs auspices entre lui et Prospero, c'était un échange fructueux entre l'élève et le maître qui lui enseignait le langage et les sciences, alors que Caliban lui dévoilait et lui offrait les richesses de l'île :

... When thou cam'st first,

Thou strok'st me, and made much of me ; wouldst give me 
Water with berries in't ; and teach me how

To name the bigger light, and how the less,

That burn by day and night : and then I lov'd thee,

And show'd thee all the qualities o'th'isle,

The fresh springs, brine-pits, barren place and fertile :

Curs'd be I that did so !

(I.ii.336-41)

Caliban semble regretter ce premier élan de collaboration, et cependant il est prêt à recommencer avec Trinculo et Stephano, alors on est conduit à penser avec Prospero que l'indigène est bien un esclave né. Par esprit de vengeance, pour un verre de vin, il est prêt une nouvelle fois à aliéner sa liberté et à donner les richesses de son île: «je veux te désigner les moindres coins fertiles et te baiser les pieds ...» (II.ii.142). «Je veux te faire voir les sources, te cueillir des fruits, pêcher pour toi, t'apporter tout ton bois [...] te déterrer des truffes, te faire voir un nid de geai ...» (II.ii.154 et seq.). Malgré cette apparente entente cordiale, ni Stephano et son acolyte, ni Prospero ne réussissent à intégrer Caliban dans leur société. Shakespeare pose insidieusement le problème de l'ordre social comme il l'a fait depuis Henry VI jusqu'à Troilus and Cressida. Le désir et le besoin de liberté conduiront nécessairement à la révolte et à la haine ${ }^{17}$.

Cette révolte et cette haine naissent du sentiment de mécontentement, de frustration et d'aliénation que crée Prospero. Caliban devient un étranger dans son propre royaume : «je forme tout votre peuple», lui dit-il, «moi qui naguère étais mon propre roi ; et vous me confinez comme un pourceau en ce dur rocher, me dérobant tout le reste de l'île.» (I.ii.331-42). Le jour où Caliban prend conscience qu'il n'est plus considéré comme égal, mais comme un démon et un esclave, surtout après l'incident avec Miranda, il réagit violemment, surtout en paroles, détournant de son usage tout ce qu'il a appris : «Vous m'avez appris à parler, et tout le profit que j'en ai tiré, c'est de savoir jurer.» (I.ii.375). Ses malédictions sont parfois empreintes d'une poésie puissante qui s'inspire de la nature: «que la plus pernicieuse rosée jamais recueillie par une mère [...] s'abatte sur vous deux !», dit-il à Prospero et à Miranda, «que le vent du sud-ouest souffle sur vous et vous couvre de pustules (I.ii.323 et seq.) [...] Que s'abattent sur vous tous les charmes de Sycorax, crapauds, scarabées, chauvessouris ...» (I.ii.341-2). Plus tard, harassé et fatigué de porter le bois, il poursuit ses imprécations: "Que tous les miasmes que le 
soleil aspire dans les marais, dans les bourbiers, dans les bas-fonds s'abattent sur Prospero pour infecter chaque pouce de son corps.» (II.ii.1-3).

Caliban sait aussi être fin politique, il sait utiliser l'ambition de Stephano et de Trinculo, et il est prêt à s'allier avec ses nouveaux dieux pour chasser l'usurpateur. Il a parfaitement assimilé la devise selon laquelle il faut diviser pour régner, et il cherche habilement à opposer les deux comparses; il fait monter les enchères, se promettant au plus offrant: (à Stephano) «Comment va Ton Honneur? Laisse-moi lécher ton soulier. Pour celui-là (Trinculo), je ne veux pas le servir, il manque de courage.» (III.ii.22 et seq.). Lorsque Trinculo mord à l'hameçon, Caliban attise le feu de la discorde entre les deux complices. Tout ceci pour les enfermer dans une logique de conspiration contre leur maître commun, Prospero : «ce tyran, ce sorcier qui, par ses artifices, lui a volé son île et l'a réduit en esclavage.» (III.ii.39-40). Dans une certaine mesure, le bouffon et l'ivrogne sont les agents de Caliban, comme Ariel est l' "esprit" de Prospero, l'un promettant un pouvoir illusoire, et l'autre une liberté hypothétique. Le meurtre de Prospero est minutieusement prémédité, car Caliban connaît les habitudes de son maître qui a coutume de faire la sieste - cliché très colonial - dans la torpeur des après-midis sinon tropicaux, du moins méditerranéens, jouissant sans doute du dernier confort occidental, avec tous les merveilleux ustensiles (III.ii.89) qui semblent tant étonner et séduire Caliban. Aujourd'hui la télévision, le réfrigérateur et l'air conditionné auraient le même effet. Caliban se complaît dans la description anticipée du meurtre de son ennemi : «lui faire sauter la cervelle, lui marteler le crâne avec une bûche, l'éventrer à l'aide d'un pieu, lui trancher la gorge avec un couteau.» (III.ii.80 et seq.). Shakespeare nous propose ici une image très conventionnelle du barbare sanguinaire. Un seul de ces moyens aurait suffi à éliminer son ennemi, mais Caliban va encore plus loin, et il insiste pour qu'on s'empare d'abord et surtout des livres de Prospero, les livres de magie qui sont la source de tous ses pouvoirs : «Mais souviens-toi d'abord de faire main basse sur ses livres ; sans eux, il n'est pas plus malin que moi et n'a plus un seul esprit à ses ordres.» (III.ii.84 et seq.). Enfin, ce rêve d'assassinat est parachevé dans la salissure qu'il promet à Prospero jusque dans sa descendance et à sa prospérité à travers Miranda. La haine de Caliban vient en partie de son désir inassouvi pour Miranda, désir interdit par un code moral et social qui lui est étranger. Il 
commet une triple transgression : il est incapable de maittriser ses désirs et ses pulsions, ensuite il imagine pouvoir passer outre les barrières sociales, et enfin et surtout il prétend aimer une femme blanche à une époque où le pouvoir noir n'avait pas encore fait triompher le slogan: "black is beautiful". Dans l'incapacité de posséder Miranda, et peupler son île de petits "Caliban", il se réjouit à l'idée que Stephano - qu'il appelle dieu pour le flatter, mais qu'il juge sans doute dès le début comme un être assez abject - que ce Stephano donc la mette dans son lit et lui donne une superbe portée de petits blancs probablement dégénérés si on en juge par le degré d'imprégnation alcoolique du géniteur :

Ay, lord ; she will become thy bed, I warrant,

And bring thee forth brave brood.

(III.ii.102-3)

Il est peut-être permis de compatir à l'éventuelle frustration de Miranda, d'autant plus qu'elle est citée à la suite des "ustensiles", et apparaît comme l'ultime agent de vengeance qu'il cherche à mettre au service de Stephano.

Le projet du front de libération de l'île échoue lamentablement, les conspirateurs pataugent dans la vase et le purin où ils ont perdu leurs bouteilles en même temps que leur honneur (IV.i.208). Quand ils arrivent devant la grille de Prospero, ils découvrent ses habits dans une malle, et se déguisent en ducs; la tragédie potentielle tourne à la farce dès l'instant où ils se contentent de revêtir les oripeaux du pouvoir. Ils sont ensuite chassés comme des bêtes malfaisantes par Ariel et ses chiens (IV.i.250 et seq.). La cruauté de la poursuite retourne la situation en faveur de Caliban qui suscite pitié et compassion face à l'insensibilité du colon Prospero.

En dépit de ses calculs, Caliban agit la plupart du temps selon son instinct, tantôt c'est son attirance vers Miranda, pour assurer sa descendance (I.ii.349) ${ }^{18}$, tantôt c'est sa générosité spontanée avec Prospero et Stephano, ou encore sa naïveté face aux forces qui le dépassent. Malgré sa servitude, imposée ou consentie, Caliban est un être libre dans ses pensées et dans ses croyances. Fils d'une sorcière et d'un démon, il vivait et continue à vivre dans l'état de nature. Sa connivence avec les hommes dépravés venus du monde civilisé n'est qu'un avatar. Mi-homme mi-bête, cet état équivoque lui permet de vivre heureux dans un milieu naturel qu'il connaît bien et qu'il aime. Dans sa naïveté de primitif, dans son innocence 
(le mot étant pris dans son acception usuelle et étymologique), Caliban est le symbole de toute la poésie de l'île, tous ses sens sont en éveil, il connaît les coins et les recoins de ce demi-paradis. Comparé à Ariel, certes capable de verser une petite larme, et à Prospero absorbé par ses livres de magie appliquée, Caliban a compris depuis toujours, et sans doute avant son maître, qu'il «est de la même étoffe que celle dont les songes sont faits» ${ }^{19}$. Même esclave, il reste maître de ses rêves et vit libre, en harmonie avec la nature :

... the isle is full of noises,

Sounds and sweet airs, that give delight, and hurt not.

Sometimes a thousand twangling instruments

Will hum about mine ears; and sometimes voices,

That, if I then had wak'd after long sleep,

Will make me sleep again : and then, in dreaming,

The clouds methought would open, and show riches

Ready to drop upon me; that, when I wak'd,

I cried to dream again.

(III.ii.133-41)

Quand son instinct le trompe, quand il prend des ânes pour des dieux, c'est à cause de la "dive bouteille", et du vin de Xérès. Remède universel, recours souvent utilisé par les colons, l'alcool agit de façon perverse, et Caliban s'agenouille devant ceux qui lui procurent cette liqueur céleste. Les dieux étaient déjà tombés sur la tête.

$*$

L'ambiguïté du concept de "nature" se manifeste dans le personnage de Caliban. Nous venons de souligner son comportement brutal, instinctif, sauvage et en même temps sa perception esthétique naturelle. Tout ceci s'oppose à l'univers civilisé de Prospero qui, au machiavélisme et au cynisme du pouvoir, ajoute lui aussi une part d'irrationnel, fruit de la magie. Shakespeare semble s'interroger sur les liens qui unissent Caliban à la nature et par conséquent sur la valeur de cet état naturel. Sur l'ambiguïté du caractère primitif de Caliban, sur la conception floue et contradictoire qu'on pouvait se faire du "sauvage" à l'époque élisabéthaine, Frank Kermode, dans son introduction à The Tempest ${ }^{20}$, traite le sujet de façon subtile et convaincante. Je soulignerai encore une fois l'importance des angles d'approche par les protagonistes du drame, par Prospero et par Caliban évidemment, mais aussi par les 
personnages qu'on hésite à qualifier ici de secondaires. Trinculo s'interroge pour savoir si Caliban est homme ou poisson, s'il est mort ou vivant, et conclut tout simplement que c'est un indigène (II.ii.25). Stephano découvre un monstre à quatre pattes (II.ii.64) et à deux voix (89), autrement dit "un monstre très délicat" (II.ii.93) ; cet oxymore contient toute l'ambiguité de Caliban. Les différents statuts ou l'absence de statut de Caliban (on ne sait trop ce qu'il est : homme, poisson, démon, Indien) jettent le trouble chez le lecteur plus que chez le spectateur, car le metteur en scène doit faire un choix ${ }^{21}$.

Le statut d'Ariel est encore plus ambigu. Le plus libre des "esprits" est successivement prisonnier de Sycorax et de Prospero. Ariel, le prompt et dévoué serviteur, est aussi un mutin impertinent toujours prêt à revendiquer sa liberté et à faire appliquer à la lettre la tacite convention collective: "encore du travail! Si tu me fais peiner, permets-moi de te rappeler", dit-il à Prospero, "ce que tu m'as promis, mais n'as pas encore tenu" (I.ii.242), il veut dire sa liberté. Prospero fait clairement allusion à son ancienne aliénation, quand il servait Sycorax, et il lui rappelle de quels tourments il l'a délivré, quand il l'a découvert, piégé depuis douze ans dans le tronc éclaté d'un pin (I.ii.277) pour ne pas avoir obéi aveuglément aux ordres de l'Algérienne, la sorcière aux yeux bleus (I.ii.269). Prospero, à la fois libérateur et tyran, encore une des ambiguittés de son statut, trouve des circonstances atténuantes à son esclave : «Toi, mon esclave, c'est là le nom que tu as pris, tu fus alors son serviteur, esprit trop pur et délicat pour ses commandements trop vils et abhorrés» (I.ii.276 et seq.). C'est le nouvel arrivant, le colon, le conquérant qui "dit le droit", et sépare le bien du mal ; le code pénal est désormais celui de Prospero. A la moindre tentation de rébellion, il sera aussi dur et même pire que Sycorax : «si tu murmures encore, moi je veux fendre un chêne et te clouer au cœur noueux, tant que tu aies hurlé au long de douze hivers» (I.ii.294). Il ne reste donc à Ariel qu'à se soumettre (297). Ariel, l' "esprit" délicat (c'est le même adjectif qui est employé pour qualifier le monstre Caliban), l' "esprit" de beauté et de lumière qui était étouffé par les forces de l'obscurantisme et de la superstition, change seulement de maître. Il sert un monarque éclairé mais néanmoins tyrannique. Il est l'allié objectif, l'agent du néocolonialisme, le collaborateur du nouvel occupant, peut-être parce qu'ils ont un ennemi commun : Caliban, et peut-être aussi parce qu'il se laisse tenter par la carotte de l'indépendance et de la liberté 
que Prospero lui agite devant le nez jusqu'au dernier acte. Combien de fois il répète à l'impatient "esprit": «oui tu seras libre, libre comme l'air, mais, avant, il faut m'obéir et accomplir des tâches toujours renouvelées.» (I.ii.504; IV.i.260; V.i.86, 97, 242). Pourtant c'est apparemment à regret que Prospero rend aux éléments son très cher Ariel, son petit poulet (V.i.315), son si charmant, son ardent Ariel (V.i.97, 242), lui souhaitant bonheur et liberté, ce qui peut aussi témoigner d'une cynique ironie ou d'un attachement trouble entre le maître et l'esclave. Prospero perd avec Ariel l'agent de son pouvoir magique, et rejette, en rejetant Caliban, ce qui pouvait le rattacher à la Nature, il ne lui reste pas d'autre choix que celui de retoumer dans le monde civilisé.

L'approche contemporaine du colonialisme et de l'anticolonialisme nous empêche souvent d'avoir une vision objective du problème, dans la mesure où elle est possible. Il serait aussi faux de dire qu'à l'époqque élisabéthaine et jacobéenne, la question était éludée, et à ce propos Montaigne et Bacon apportèrent leur témoignage en leur temps. Cependant, des pièces comme The Travailes of Three English Brothers, les rapports officiels, ou les récits des voyageurs font plus que l'apologie de la force comme moyen de s'implanter dans une colonie, l'évangélisation et le progrès n'étant qu'un alibi pour mieux exploiter les ressources et tirer de plus grands profits du commerce avec les indigènes. Ces derniers trouvent d'ailleurs leur place dans ce qu'on a appelé la hiérarchie de la chaîne des êtres. Leur qualité d'être humain est mise en question, homme, poisson, démon, monstre, ceci a déjà été souligné plus haut, et ce qui pourrait arriver de mieux à Caliban serait de finir comme une bête curieuse sur une place publique. D'abord considéré comme un être récupérable, il n'est plus, après avoir tenté de séduire la blanche Miranda, qu'un animal domestique juste bon à transporter des charges de bois (car le climat lui-même est colonisé, anglicisé), et après le complot qu'il a ourdi, il est pourchassé comme un vulgaire gibier par une meute de chiens. L'allégorie, ou simplement la fable, pourrait bien illustrer de manière anachronique l'attitude des Anglais en Amérique, chassant et exterminant les Indiens rebelles, et exploitant les noirs comme des animaux domestiques, allant même jusqu'à encourager leur reproduction. Mais Prospero ne vient pas dans l'île pour conquérir, pour trafiquer ou pour évangéliser, c'est un naufragé, un exilé, et ce ne sont pas les livres saints qu'il rapporte dans son esquif, mais des livres de 
magie. Ainsi, le problème est peut-être ailleurs. Le fait que Caliban soit imperméable à la civilisation - bien qu'il parle assez bien pour jurer comme un véritable Anglais - c'est l'aveu d'un échec, celui de la raison incapable de maîtriser l'instinct. C'est l'échec de la spiritualité incapable de maîtriser la chair qui se complaît dans la fange et se perd dans l'alcool, dans une soif de liberté qui ne peut être qu'anarchique. Prospero libère Ariel et renvoie Caliban dans sa grotte, il renonce à son œuvre d'éducation comme il a déjà renoncé à son œuvre de colonisation. Ce faisant, il semble reconnaître la nécessaire présence du "mal", de la "nature", et de l'instinct dans "la complexité de la vie"22. Mais dans une perspective "pré-coloniale", j'allais dire édénique, le mal n'a aucun sens, le mal est un produit d'importation, une notion étrangère, et Prospero le remporte avec lui dans ses bagages. Comparé au trio dérisoire composé de Stephano, Trinculo et Caliban, le vrai conspirateur est Antonio. Bien qu'il soit inclus dans l'amnistie générale, il symbolise la permanence du mal civilisé, et reste une menace pour l'ordre soi-disant retrouvé. Prospero ferme les yeux sur la réalité et a beaucoup de mal à réintégrer son ancien univers. Il est certainement plus à l'aise avec sa baguette magique qu'avec sa couronne de duc. De même quand il découvre Miranda et Ferdinand jouant aux échecs, il semble ne pas vouloir entendre sa fille qui remarque le jeu trompeur de son fiancé : «Doux seigneur, vous trichez» (V.i.173-6). Peut-être Prospero estil arrivé à une sorte de lucidité stoïque : il comprend qu'Antonio pourra recommencer ses intrigues, que Ferdinand, en qui il mettait pourtant toute sa confiance, est lui aussi capable de tricher, et que Caliban lui échappe. Celui-ci pleure du regret de ses rêves (III.iii.151), alors que Prospero, comme Hamlet appelle le sommeil et la mort après une pauvre vie qui est «un îlot dans le sommeil qui nous entoure» (IV.i.156). On comprend mieux alors que Prospero ne manifeste pas trop d'enthousiasme pour retourner dans son duché. Le monde civilisé n'est sans doute pas aussi merveilleux qu'on pourrait le croire, et quand Miranda s'extasie avec la candeur de l'innocence sur la «splendide humanité, beauté de ce monde nouveau qui peut nourrir des être si parfaits» (V.i.184), il ne faut pas oublier la dimension ironique, et il est bon de rappeler que c'est là précisément que Aldous Huxley a trouvé le titre de son roman philosophique Le meilleur des mondes, dont le héros est justement Monsieur Sauvage. 
Il serait hasardeux de conclure à la réconciliation des deux mondes, celui de l'Italie de Prospero, et celui de l'île de Caliban. Chacun a sa vérité, et Shakespeare semble se ranger du côté de Montaigne qui souligne que «chacun appelle barbarie ce qui n'est pas de son usage ${ }^{23}$. L'un voit des démons où l'autre croit découvrir un âge d'or. Quand à la réconciliation entre la "splendide humanité" et le monde de la nature, il est difficile d'y croire. L'île, cette utopie, ce lieu imprécis, ce "non-lieu" entre Naples et Tunis a une double fonction: elle permet à Gonzalo d'imaginer et de projeter vers l'avenir une société parfaite, et elle permet au Masque de rappeler un âge d'or disparu. «La redécouverte du passé est une invitation pour les rescapés du naufrage à prendre conscience de ce que pourra être l'avenir, une fois la réconciliation accomplie. Mais l'art de Prospero, qui peut faire se dresser les morts de leurs tombeaux, est impuissant à réformer les hommes» 24 . Il y a aussi l'autre volet où est brossé un tableau critique de la société civilisée, avec ses conspirateurs, sa corruption, ses tares et la fragilité de son équilibre. En effet, tous ont plus ou moins failli à leur mission, tous ont plus ou moins transgressé les codes: Alonso a marié sa fille à un "Africain", et Sebastian fait bien comprendre que la perte de son fils est la punition de cette faute :

Sir, you may thank yourself for this great loss, That would not bless our Europe with your daughter,

But rather lose her to an African.

(II.i.119-21)

Prospero a délaissé son duché pour s'adonner à des études ésotériques, et l'expérience de l'exil ne semble pas lui avoir apporté beaucoup de sagesse. Certains ont voulu voir dans The Tempest le thème de «la réconciliation, de la restauration d'un ordre perdu, de la confiance faite à la vie après l'expérience de la souffrance et du repentir» ${ }^{25}$. Mais si le bien triomphe, on peut s'interroger sur la nature de ce "bien", et sur les moyens de sa victoire. «Il ne s'agit pas de la restauration d'un ordre naturel dont la pièce postulerait qu'il existe en lui-même et en dehors d'elle» ${ }^{26}$. La violence et l'horreur, sont toujours présentes, même derrière le masque illusoire de la magie.

L'ambiguïté du colonialisme et des valeurs du monde civilisé s'accompagne d'une mise en question permanente du réel. Les personnages s'interrogent sur la nature de ce qu'ils voient ou perçoivent, Ferdinand demande d'où vient la musique qu'il entend 
(I.i.390), les naufragés s'étonnent de découvrir que leurs vêtements sont aussi secs et aussi frais qu'au premier jour, malgré le naufrage et l'eau de mer (II.i.61), ils sont surpris de ne pas voir les bêtes sauvages qu'ils croient entendre (II;I.305-320), leurs sens sont constamment mis à l'épreuve et abusés. L'illusion est effectivement représentée sur scène avec le festin qui est amené et soudain disparaît (III.III.20-52) ; il faut rappeler que ce procédé si spectaculaire avait déjà été éprouvé dans le Doctor Faustus de Marlowe. Il y a un rapport constant entre le merveilleux et le véridique si bien que tout devient relatif, et que les personnages peuvent passer soudain de la conviction au doute ou du scepticisme à la crédulité comme Sebastian qui, après ce qu'il a vu, est prêt à croire les choses les plus extraordinaires :

... Now I will believe

That there are unicorns; that in Arabia

There is one tree, the phoenix' throne; one phoenix

At this hour reigning there.

(III.iii.21-4)

Je donnerai un dernier exemple de cette pluralité et de ce déplacement permanent des perspectives : la tempête. C'est d'abord une vraie tempête, un vrai naufrage de théâtre, et Ariel précise bien aux spectateurs, mais non aux protagonistes, qu'il s'agit d'une fausse tempête, d'une simple mise en scène, et jusqu'à la fin elle devra être perçue au premier degré par les vrais faux naufragés. Les personnages, sur scène, s'interrogent sur la nature de ce qu'ils voient alors que le spectateur voit non seulement avec ses propres yeux, mais aussi avec ceux de Prospero par l'intermédiaire d'Ariel. Prospero est le metteur en scène de toute l'action jusqu'à la réconciliation finale qu'il organise méticuleusement en excluant toute intervention providentielle. Prospero, «le maitre du discours et des événements est aussi la maître du sens» ${ }^{27}$.

The Tempest est un récit qui s'inscrit dans une mythologie dont la cohérence satisfait plus l'imaginaire que la raison, l'éthique ou les sciences politiques ${ }^{28}$. Dans l'histoire du colonialisme, la pièce peut tenir un rôle important, elle anticipe plus qu'elle n'explique, elle pose plus de problèmes qu'elle n'en résout. Prospero réorganise le passé à sa manière et prépare Miranda à un monde où le mal a sa place. Il tente de créer un nouveau mythe, au prix de quelques manipulations, cherchant une logique a posteriori, un récit ancien donnant un sens au récit nouveau. Miranda a d'ailleurs de la peine à suivre le dédale de ces fables du temps passé, et son at- 
tention faiblit souvent malgré les rappels de son père : «Que voistu d'autre dans l'obscur arrière-fond, dans l'abîme du temps? Si tu peux rappeler quelque chose antérieure, rappelle-toi comment tu vins ;》 (I.ii.49-52). Prospero n'est pas à la recherche d'un nouveau monde, il est à la recherche de son propre passé, et c'est bien là le but final du voyage. Lui et les autres, accompagnés des nouveaux époux, symboles de l'avenir, tous, sauf Caliban, n'ont qu'un seul but : retourner vers l'Italie, vers l'ancien monde. «L'Age d'or ne se trouve pas sur les Iles fortunées, mais dans les spectacles, les visions ou les vœux des hommes» ${ }^{29}$. Shakespeare nous suggère que l'Eldorado ne se trouve pas sur une île, mais dans les désirs et dans les rêves, dans l'illusion si bien représentée par le Masque, et dans les constructions utopiques de Gonzalo.

Si les colonies et la colonisation sont bien présentes dans The Tempest, elles sont ausi un outil de réflexion sur les rapports entre les hommes, et entre les hommes et la nature. L'île est un mirage plus qu'une terre à coloniser, c'est un moment de rêve, c'est un mode d'illusion qui doit permettre à chacun de s'interroger sur la réalité des choses: «c'est le dédale le plus étrange qu'homme ait jamais foulè» (V.i.242) dit Alonso. C'est un univers d'incertitudes où les sens sont mis en doute :

GONZALO

... Whether this be

Or be not, l'll not swear.

PROSPERO You do yet taste

Some subtleties o'the isle, that will not let you

Believe things certain.

(V.i.123-5)

Comme l'écrit Richard Marienstras : «Ce qu'on découvre au terme du voyage, ce ne sont pas vraiment d'autres mondes : ce sont des moments du nôtre. Le voyage réunit ce qui était disjoint, le lointain devient proche, si dangereusement proche qu'il faut toujours réintroduire, entre le monde lointain et celui de la civilisation chrétienne, des distances familières ${ }^{30}$.

L'épaisseur de l'histoire nous empêche de faire des comparaisons entre la notion même de colonialisme qui pouvait prévaloir au temps de Shakespeare et les acceptions contemporaines, nécessairement différentes selon les points de vue. On se méfiera donc 
de toute généralisation et de toute actualisation abusives. Le jeu des ambiguïtés met en évidence la relativité des valeurs. Shakespeare nous suggère qu'on est toujours l'esclave d'un autre ou de soi-même, mais que la liberté, notion toute relative, peut aussi finir par être gagnée. The Tempest reflète l'interrogation de la conscience occidentale sur l'exploitation coloniale qui se développe surtout avec l'arrivée des Européens en Amérique. Bien qu'il soit cruel, autoritaire, orgueilleux, méprisant, Prospero est souvent considéré, même si c'est une hypothèse spécieuse et dérisoire, comme le porte-parole de Shakespeare. Le Duc de Milan est le metteur en scène, le magicien capable d'agir sur les choses, sur les êtres et il est surtout le maître des mots, c'est-à-dire le poète, le dramaturge. Il est aussi le politique, il combat tous les désordres, publics ou privés, il prône la hiérarchie, la discipline, et il restaure l'ordre ancien. Même si Prospero tient un discours conservateur et réactionnaire, il n'est qu'un masque. D'ailleurs, dans sa lutte farouche contre la nature et l'instinct incarnés par Caliban, il reconnaît la part de l'irrationnel. Le pouvoir lui vient des livres de magie, tout comme celui de la sorcière Sycorax venait du démon; blanche ou noire, la magie est toujours la magie, et le pouvoir n'est qu'illusion. A la fin, le colon retrouve son univers civilisé où la tricherie et les intrigues sont toujours possibles, alors qu'Ariel vole vers la liberté, et que Caliban garde intacte sa nature sauvage. Shakespeare afflige ce monstre de trop de défauts humains pour que nous puissions croire qu'il n'est ni chair ni poisson, il est homme avant tout, il est notre frère. Il est sans doute, en partie, le reflet de la vision du sauvage que pouvait avoir un Anglais à l'époque des Stuarts, mais il possède une dimension pathétique et poétique, une dignité qui nous le rendent plus cher et plus sympathique qu'Ariel qui, par ses compromissions, fait le jeu de l'occupant. Le combat de Prospero est douteux, sa victoire ambiguë, mais ses discours comme ses actions sont loin de légitimer le colonialisme, et posent plus de questions qu'ils n'appellent de réponses.

Jean-Pierre VILLQUIN

Université de Nantes 


\section{NOTES}

1. «The revisionist impulse has been one of the most salutary in recent years in correcting New Critical "blindness" to history and ideology. In particular it has revealed the ways in which the play has been "reproduced" and drafted into the service of colonialist politics from the nineteenth century through $G$. Wilson Knight's twentiethcentury celebration of Prospero as representative of England's «colonizing, especially her will to raise savage peoples from superstition and blood-sacrifice, taboos and witchcraft and the attendant fears and slaveries, to a more enlightened existence.» Cette citation est empruntée au remarquable article de Meredith Anne Skura, "Discourse and the Individual: The Case of Colonialism in The Tempest", Shakespeare Quarterly, vol. 40, Spring 1989, $\mathrm{n}^{\circ} 1$, p. 46. L'auteur analyse les différentes écoles critiques, et dresse dans son introduction (pp. 4247), un inventaire des publications récentes sur le sujet.

2. «Caliban is a grotesque figure, a grim caricature of the good savage depicted by Montaigne». François Laroque, "Cannibalism in Shakespeare's imagery", Cahiers Elisabéthains, n" 19, Montpellier, avril 1981, p. 34.

3. Voir l'intéressant article de Alden T. Vaughan, "Shakespeare's Indian: The Americanization of Caliban", dans Shakespeare Quarterly, vol. 39, Summer 1988, $\mathrm{n}^{\circ} 2$, pp. 137-153.

4. Le texte utilisé est The Tempest, ed. by Frank Kermode, The Arden Shakespeare, 1954, rep. 1989, Routledge, London and New York.

Les citations en français sont empruntées pour la plupart à la traduction de Pierre Leyris, Shakespeare, Euvres complètes, Le Club français du livre, Paris, 1968, vol. XII.

5. Day, Rowley et Wilkins, The Travailes of Three English Brothers (1606/7).

6. «I like a Plantation in a Pure Soile; that is, where People are not Displanted to the end to Plant in Others. For else, it is rather an Extirpation then a Plantation.» Bacon, Essays, M. Castelain, Aubier, Paris, 1948, pp. 176 et 178.

7. Henri Fluchère, dans Essais critiques sur l'œuvre dramatique, Shakespeare, la Pléiade, Gallimard, Paris, 1959, p. 583.

8. Richard Marienstras, Le Proche et le lointain, Les éditions de minuit, Paris, 1981, p. 248.

9. Samuel Purchas (1575-1626), vol. IV de sa compilation; Hakluytus Posthumus or Purchas his Pilgrimes, contayning a History of the World in Sea Voyages and Lande Travells, by Englishmen or others, 4 vol. 1625. «L'ouvrage veut être une suite aux Navigations de Hakluyt.»Voir H. Fluchère, op. cit., p. 583. 
10. Thomas Russel (1570-1634). Voir F. E. Halliday, $A$ Shakespeare Companion, G. Duckworth \& Co. Ltd., London, 1950, rep. 1968 , p. 427.

11. Voir H. Fluchère, op. cit., p. 585.

12. Montaigne, Essais, I, ch. xxxi, La Pléiade, Gallimard, Paris, 1975, p. 204.

13. «If you Plant where Savages are, doe not onely entretaine them with Trifles and Gingles, But use them iustly and gratiously, with sufficient Guard neverthelesse.» Francis Bacon, Op. cit., p. 182.

14. «Sur l'assimilation du Noir à l'animal domestique, voir l'important ouvrage de Winthrop D. Jordan, 1968, p. 1-91, (III).» Note de R. Marienstras, op. cit., p. 244.

15.... this thing of darkness, I Acknowledge mine. (V.i.275-6).

16. Vendredi sera lui aussi pétrifié par le fusil de Robinson Crusoe qui peut tuer à distance. Dans The Travailes, pièce déjà citée, on voit le Sophi de Perse bouche bée devant les canons que lui a présentés Sir Anthony Shirley :

... your warres are royall,

So ioyn'd with musicke that even death itselfe

Would seeme a dreame : your instruments dissolve

A body into spirit but to heare

Their cheerful Clamours; and those your Engins

(We cannot give their proper Character)

Those lowd tongues that spit their spleene in fire ...

John Day, Works, R. Jeffs, ed. Holland Press, London, 1963, p. 324.

17. Voir H. Fluchère, op. cit., p. 593.

18. Thou didst prevent me: I had peopled else

This isle with Calibans. (I.ii.352-3)

19. Pros. ... We are such stuff

As dreams are made on; and our little life

Is rounded with a sleep. (IV.i.156-8)

20. The Tempest, op. cit., introduction, pp. xxxiv-xliii.

21. Voir l'article de Victor Bourgy, "On Caliban's Nature", Cahiers Elisabéthains, $n^{\circ}$ 43, April 1993, pp. 35-42.

22. «Peut-être que Prospero, qui, libérant Ariel, se contente de renvoyer Caliban dans sa grotte avec promesse de pardon pour bonne conduite, reconnaît implicitement la nécessité de cette présence dans la complexité de la vie.» H. Fluchère, op. cit., p. 593.

23. Montaigne, op. cit., I, xxxi, p. 203. Voir également le commentaire de R. Marienstras : "Ce qu'on découvre au terme du voyage, ce ne sont pas vraiment d'autres mondes: ce sont des moments du nôtre. Le voyage réunit ce qui était disjoint, le lointain devient proche, si dangereusement proche qu'il faut toujours réintroduitre, entre le monde lointain et celui de la civilisation chrétienne, des distances familières.» op. cit., p. 243. 
24. M.-T. Jones-Davies, Shakespeare, le théâtre du monde, Balland, Paris, 1987, p. 202.

25. Voir H. Fluchère, op. cit., p. 588.

26. R. Marienstras, op. cit., p. 269.

27. R. Marienstras, op. cit., p. 269.

28. Voir l'article de Raymond Gardette, "Ariel et Caliban: l'imaginaire monstrueux dans La Tempête de W. Shakespeare", Monstres et prodiges au temps de la Renaissance, Centre de Recherche sur la Renaissance, directeur de la publication M.-T. Jones-Davies, J. Touzot, éd. Paris, 1980, pp. 69-80.

29. R. Marienstras, op. cit., p. 268.

30. R. Marienstras, op. cit., p. 243. 